\title{
ENERGY-BALANCED ON DEMAND CLUSTERING ALGORITHM BASED ON LEACH-C
}

\author{
KHAMISS.A.A1, ZHANG Baihai1, CHAI Senchun*1and CUI Lingguo1 \\ 1School of Automation, Beijing Institute of Technology, Beijing, 100081, China
}

\begin{abstract}
As the use of Wireless Sensor Networks (WSNs) has grown enormously, the need for energy-efficient management has also risen. With advances in ubiquitous computing environment, WSNs have been broadly studied and many energy-efficient routing protocols had been proposed. LEACH (Low Energy Adaptive Clustering Hierarchy) is a popular cluster-based protocol, which provides distributed adaptive clustering and periodic cluster head $(\mathrm{CH})$ selection rotation. As extension to LEACH, LEACH-C (LEACH Centralized) was proposed, in which the energy is utilized to select CH. However, both can't guarantee cluster head distribution, in addition to considerable periodic clustering overhead. Furthermore, network topology change is a critical characteristic that has influence on communication path and load distribution among nodes. To resolve such problems, Energy-Balance on Demand Clustering Algorithm Based on LEACH-C is proposed. The algorithm adopts centralized cluster formation and distributed CH selection methods. Minimum energy clustering is used to divide the network into clusters, while energy and total communication distance are considered as secondary criteria to select optimal CH. From simulation results the proposed algorithm outperforms $\mathrm{LEACH}-\mathrm{C}$ in life time, stability period and performance efficiency.
\end{abstract}

\section{KEYWORDS}

K-mean, total communication distance, Low level energy \& Cluster energy

\section{INTRODUCTION}

Wireless Sensor Networks (WSNs) typically consist of a large number of low-cost, low-power and multifunctional wireless sensor nodes. Nodes are equipped with sensing, communication and computation capabilities, where they communicate via a wireless medium and work collaboratively to accomplish a common task [1]. The basic philosophy behind WSNs is that, while the capability of each individual node is limited, the aggregate power of the entire network is sufficient for the required mission [2]. WSNs had been deeply studied in last decades due to their wide range of applications in different fields including environmental management, healthcare, security and surveillance, industrial control, military applications and more complicated applications are expected in the future [3-6]. In most applications, nodes are deployed randomly, and once deployed they must be able to autonomously organize themselves into a network. Generally WSNs are characterized with high density of deployed nodes, while the nodes themselves are unreliable and restricted in power, computation and storage resources. And due to these characteristics, suitable network routing protocols are required to implement various network control and management functions. The way of finding a path from source node to the destination is known as routing protocol; therefore, routing protocols highly affect network life 
International Journal of Wireless \& Mobile Networks (IJWMN) Vol. 7, No. 1, February 2015

time and efficiency. The main objective of routing protocols is to improve efficiency and extend life time considering the nodes constraints, network limitations, sensing field nature and application requirements. Hence, selecting optimal path, forming balanced clusters, minimizing control overhead, and balancing energy load to reduce communication overhead are challenging issues of routing protocols [7]. Clustering is one of the most popular techniques used in routing protocols. Cluster-based routing is an efficient in reducing energy consumption within cluster by decreasing the messages sent to the BS. In cluster-based routing, nodes are partitioned into small groups called clusters, for fussing data via efficient network organization [8]. Each cluster has a cluster head that controls the data gathering and aggregation process after receiving data from members. Clustering in WSNs improves the scalability of the network, because clustering minimizes the need for central organization and promotes local decisions. The major benefits of clustering in WSNs are: (1) provides the spatial reuse of resources to increase system capacity. (2) Routing information of a cluster is shared with only other cluster heads or gateways. This reduces the number of transmission performed to distribute routing information. With cluster structure used in WSNs, the local changes need not to be reflected to entire network. This reduces the information processed by nodes and data stored in nodes [9]; therefore, many researches had been conducted on cluster-based routing protocols [10-17]. Most popular and classic one is LEACH [10]; the objective of LEACH is to minimize energy dissipation. LEACH has distributed coordination and control mechanisms for cluster setup and operation process. CHs are selected randomly, and rotated periodically in consecutive intervals known as rounds. Due to random selection of $\mathrm{CHs}$, a node with low energy or located far away may be selected as $\mathrm{CH}$, causing uneven energy loss and forming blind spot. To improve energy efficiency, LEACH-C [10] was proposed, which utilizes energy to select $\mathrm{CH}$. Random or energy-based selection of CHs causes' skewed distribution of clusters, i.e. $\mathrm{CH}$ may be located at the edge of the field or adjacent nodes could be cluster heads, then other nodes may consume considerable energy to reach $\mathrm{CH}$. Also the crucial problem of network stuck may be faced due to very low nodes energy. Another critical source of energy consumption is the periodic clustering overhead. Also, the steady-state duration represents a critical factor that causes data loss when $\mathrm{CH}$ dies out during this period. In this paper, we propose energy-balanced on demand clustering algorithm based on LEACH-C, which adopts a centralized cluster construction and distributed $\mathrm{CH}$ selection approaches. Nevertheless, the algorithm relates the steady-state duration to node energy status to avoid data loss due to energy depletion of $\mathrm{CH}$ or member node. After receiving location and energy information from nodes, the BS forms the clusters via iterative algorithm, computes distance to neighbours for each node. Then the BS broadcasts the clustering information, distance to neighbours, and total energy to all nodes. Upon receiving this information every node can decide whether to become a $\mathrm{CH}$ within its cluster or not. The proposed algorithm outperforms LEACH-C and demonstrates the advantage of balanced energy consumption overall the network and reduction of control overhead. The rest of this paper is organized as follows; section II represents the related works, while section III explains the network energy model. Section IV discusses the proposed algorithm in details. Section V provides the simulation and discussion of the results. Finally, section VI concludes the paper with final remarks and future works.

\section{RELATED WORKS}

Many and different cluster-based energy-efficient routing protocols have been proposed for WSNs [10-21]. In this section abbreviated overview of some cluster-based protocols, along with their limitations will be presented. In [10] the first hierarchical clustering algorithm LEACH was 
International Journal of Wireless \& Mobile Networks (IJWMN) Vol. 7, No. 1, February 2015

proposed. LEACH is a cluster-based protocol that randomly selects $\mathrm{CHs}$, where the number of $\mathrm{CHs}$ is adaptive and the selection process is dynamically rotated to evenly distribute load. LEACH suffers from random selection of $\mathrm{CHs}$ and it works best only if the energy of nodes is uniform. There is no any certainty about the distribution of $\mathrm{CHs}$ throughout the network, and the idea of dynamic clustering brings extra overhead. In order to distribute $\mathrm{CHs}$ evenly over network, LEACH-C [10] was proposed as an extension to LEACH, the idea is that, the nodes inform the BS about their energy and locations, and the BS calculates the average energy of the network, then the nodes with energy less than average energy will be excluded from competition. The BS runs optimization algorithm to select $\mathrm{CHs}$ and forms clusters by adding the nodes to their closest $\mathrm{CH}$. The problem is that, if the high energy nodes are located at the edges, the same drawbacks of LEACH will be met. Moreover, LEACH-C requires sending of location and energy information at the beginning of each round. E-LEACH, improves the $\mathrm{CH}$ selection by making the residual energy of the node as the main metric which decides whether the nodes turn into CHs or not after the first round. In the first round all nodes have the same probability to become $\mathrm{CH}$, i.e. randomly as LEACH, in the next rounds the residual energy of each node is different and it is taken into account. That means, nodes have more energy, irrespective of their locations, will become CHs rather than nodes with less energy [11]. MR-LEACH multi-hop routing with LEACH in [12], partitions the network into layers of clusters based on distance to the BS. MR-LEACH outperforms LEACH-C in energy consumption, but the $\mathrm{CH}$ selection in a layer only depends on energy without considering the distances among CHs. In [13] distance-energy cluster structure algorithm (DECSA) was proposed, where the distance and energy of nodes are considered. DECSA reduces energy consumption due to multi-hop communication between $\mathrm{CHs}$ and BS. Enhanced developed distributed energy-efficient clustering (EDDEEC) was proposed in [14], it selects $\mathrm{CHs}$ depending on nodes energy level. The approach dynamically changes the probability of nodes to selected nodes as $\mathrm{CHs}$ in a balanced way to distribute the energy among nodes. In LEACH-H (Hybrid cluster head selection LEACH) the base station selects the CHs in first round. While in the followed rounds the CHs select the new CHs in their own clusters. Hybrid EnergyEfficient Distributed Clustering (HEED) is a multi hop clustering that focus on efficient clustering based on the physical distance between nodes. The scheme selects $\mathrm{CHs}$ in terms of residual energy and intra-cluster communication cost, which is useful if given node falls within the range of more than $\mathrm{CH}$ [15]. The problem in HEED is that, it is important to identify what is the range of a node in terms of its power level, as node will has multiple discrete transmission power levels. In [16], FZ-LEACH (Far-Zone LEACH) is proposed to form far-zone which is a group of nodes which are placed at locations where their energies are less than a threshold. Equalized cluster head election routing protocol (ECHERP) was proposed in [17], it aims to conserve energy via balanced clustering. ECHERP uses Gaussian elimination algorithm to model the network as a linear system, and then selects the good combinations of $\mathrm{CHs}$ to minimize transmission energy consumption and, hence, prolong network life time. In [18], the concept of secondary $\mathrm{CH}$ is introduced based on the average energy and average distance to BS. The secondary $\mathrm{CH}$ is responsible for receiving and fusing data collected from members and sending them to $\mathrm{CH}$. The $\mathrm{CH}$ is only responsible for sending data to BS. In [19], $\mathrm{CH}$ is selected based on residual energy and distance to BS. Furthermore, a hierarchical routing tree with hop count is used for data transfer. Based on Qos routing, convergent traffic routing is implemented as gradient-based protocol to improve reliability and packet delivery rate for convergent traffic [20]. In [21], depending on event driven routing, the concept of packet attribute, defined as the identifier of the data sampled by different kind of sensors or applications, is introduced. The attribute-aware data aggregation was proposed to meet the need for dynamic packets forward 
according to network state or packet types and to aggregate packets from different applications or kinds of sensors.

\section{NETWORK ENERGY MODEL}

\subsection{Radio Energy Model}

The energy and radio model adopted in this work is as discussed in [10]. To achieve an acceptable signal-to-noise ratio (SNR) in transmitting $k$ bit message over a distance $\mathrm{d}$, the energy consumed for transmission $\left(\mathrm{E}_{\mathrm{TX}}\right)$ is given by:

$$
\left\{\begin{array}{l}
E_{T X}(l, d)=l E_{e l e c}+l \varepsilon_{m p} d^{4}, \text { if } d>d_{0} \\
E_{T X}(l, d)=l E_{e l e c}+l \varepsilon_{f s} d^{2}, \text { if } d \leq d_{0}
\end{array}\right.
$$

And the energy expended by the radio to receive $l$ bit message, is given by:

$$
E_{R X}=l E_{\text {elec }}
$$

where $E_{\text {elec }}=50 \mathrm{nj}$, is the energy used to run the transceiver circuitry, $\varepsilon_{f t}=10 \mathrm{pj} / \mathrm{bit} / \mathrm{m}^{2}$ and $\varepsilon_{m p}=0.0013 \mathrm{pj} / \mathrm{bit} / \mathrm{m}^{4}$ are energies used by transmitter amplifier for short and long distances respectively and $E_{d}=5 \mathrm{nj} / \mathrm{bit} /$ signal is the energy of fusion per bit. For $d=d_{0}$, the distance threshold is given by $d_{0}=\sqrt{\frac{\varepsilon_{f s}}{\varepsilon_{m p}}}$ where $d_{0}$ is known as the crossover distance.

\subsection{Assumptions}

The following assumptions are made about the sensors and the network model:

1-The sink node is located inside the sensing field and it has unlimited resources. 2-Sensors are location-aware and energy constrained. 3-Each node has a unique identifier and it is initially able to transmit data directly to the BS. 4-A node belongs to only one cluster, but may change its belonging cluster during each round. 5-Sensors are energy-aware and energy-adaptive. 6-Links are symmetric; nodes and sink both are stationary after deployment. 7-Cluster: a mixture of single and multi-hop, no assumption about the homogeneity of node category and dispersion radius.

\section{PROPOSED ALGORITHM}

The proposed scheme is a cluster-based algorithm that modifies the clustering process, thus, both the cluster formation and the $\mathrm{CH}$ selection methods had been modified. For more energy conservation, a multi-hop routing was adopted to forward data to the BS. Clustering achieves a significant improvement in terms of energy consumption, and provides scalability. Also clustering is one of the basic approaches to control topology and design energy-efficient distributed WSNs. However, these benefits can result in extra overhead due to cluster formation's 
message exchange. In this algorithm we assumed exchanging sensor data may be an expensive network operation, but exchanging data about sensor data needs not be. Since energy is the major concern, then balanced energy consumption is important in energy conservation. In LEACH-C, the cluster heads are selected according to formula (3) and then members are added to their nearest $\mathrm{CHs}$ to construct clusters in setup phase. In the proposed algorithm, clusters are formed firstly, and then a single cluster head is selected in each cluster. The major problem of LEACH-C [10] is the energy-based selection of $\mathrm{CHs}$ (based only on energy) via running optimization algorithm as in formula (3) \& (4). Formula (3) represents the probability of node $i$ to become a $\mathrm{CH}$ and formula (4) is the expected number of $\mathrm{CHs}$.

$$
\begin{aligned}
& P_{i}(t)=\max \left\{\left(\frac{E_{i}(t)}{E_{\text {tann }}(t)}\right) k, 1\right\}, \\
& \text { where } E_{\text {totati }}(t)=\sum_{i=1}^{N} E_{i}(t) \text {, and; } \\
& E[\# C H]=\sum_{i=1}^{N} p_{i}(t) * 1=\sum_{i=1}^{N}\left(\frac{E_{1}(t)}{E_{\text {rotala }}(t)}+\ldots+\frac{E_{N}(t)}{E_{\text {toostal }}(t)}\right) * k=k
\end{aligned}
$$

Where $\mathrm{E}_{\mathrm{i}}(\mathrm{t})$ is the current energy of node $i, \mathrm{E}_{\text {total }}(\mathrm{t})$ is the network total energy, $N$ is the total number of sensor nodes, and is the number of clusters. Members are added to cluster heads via BS by using simulated annealing algorithm [22]. Another energy consumption source in LEACH$\mathrm{C}$ is that, the location and energy information of each node is required at the beginning of each round. To support both energy-efficient and wide range of connectivity, an algorithm is required to consider a node's connectivity with neighbouring nodes and its distance to the BS. In the proposed algorithm, minimum energy clustering is used to form clusters while, energy and total communication distance are considered to select CHs. Clusters are constructed in centralized method via k-mean clustering method [23, 24], and $\mathrm{CHs}$ are selected in distributed probabilistic manner. That means; each node can make its decision to become $\mathrm{CH}$ within its cluster or not in autonomous manner. As in LEACH-C, the operation of the proposed algorithm consists of setup phase and steady state phase. The cluster formation, CHs selection, and multi-hop paths establishment are done successively in setup phase. While in data transmission phase, the nodes sense and transmit data to $\mathrm{CHs}$ and then $\mathrm{CHs}$ forward data to BS after aggregation. Since the energy and performance efficiency will be achieved in cost of delay, then such algorithm is suitable in constant monitoring application scenario, where time has no high priority as application requirement property.

\subsection{Setup Phase}

Three processes are done in setup; cluster formation, $\mathrm{CHs}$ selection and multi-hop paths establishment.

\subsubsection{Cluster Formation}

At the beginning all nodes send their energy and location information to BS. Since the BS has unlimited resources, it utilizes the unsupervised clustering technique k-mean [23, 24] to divide 
the network into $k$ minimum energy clusters, where ( is defined in advance as $k p$ percent of the total number of nodes). Cluster energy is defined as the sum of distance squared from each node to its cluster center. The BS calculates the total energy of the network, the sum of distances to neighbour nodes (nodes with the same centroid) for each node and broadcasts this information and the clustering information. Therefore, each node can determine the total energy of the network, its cluster centroid, its neighbours (nodes with the same centroid), and its distance to neighbours. Upon knowing this information in addition to distance to BS, which is already calculated by the node according to the strength of signal received from BS at the beginning, the node will be capable to decide whether to become a $\mathrm{CH}$ in its cluster or not. It is the goal of the $\mathrm{K}$ - mean algorithm to find, for fixed number of clusters, a clustering that minimizes cluster energy. The algorithm aims at minimizing an objective function, which is the squared error function;

$$
J=\sum_{j=1}^{k} \sum_{i=1}^{n}\left\|x_{i}^{j}-c_{j}\right\|^{2}
$$

where $\left\|x_{i}^{j}-c_{j}\right\|^{2}$ represents chosen distance measure between a node $x_{i}^{j}$ (node $i$ in cluster $j$ ) and cluster center $C_{j}$ (center of cluster $j$ ), $J$ is an indicator of the distance of the total $n$ nodes from their respective cluster centers. The algorithm works as follows; in the beginning, determine the number of clusters $k$, the centers of these clusters (initial) might be assumed randomly or the first $k$ nodes can serve as the initial centers. $\mathrm{K}$-mean algorithm will do the following three steps until converge (iterate until stable, i.e. no object move group):

1- Determine the centroids coordinate.

2- Determine the distance of each node to the centroids.

3- Group the nodes based on minimum distance.

Figure1 summarizes the steps of k-mean. After partitioning, the BS notifies the nodes by broadcasting a message containing the total energy, distance to neighbour nodes (nodes with the same centroids) and clustering information. Every node stores this message and uses its contents to decide whether it will turn into cluster head or not. 


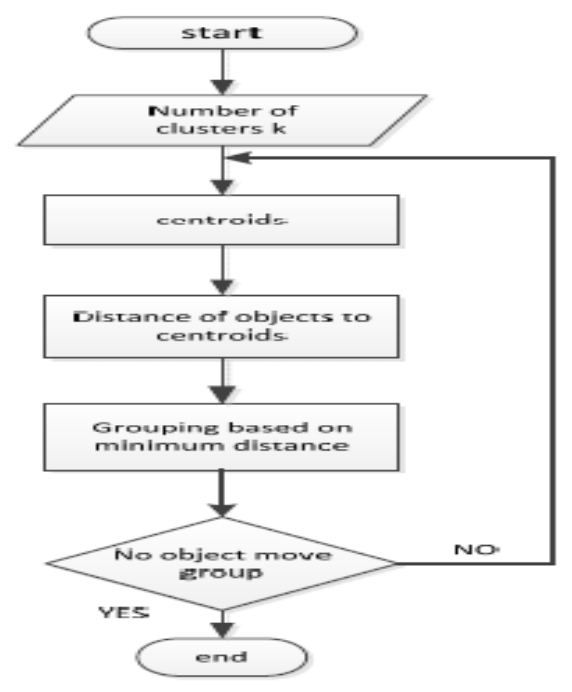

Figure 1. K-mean algorithm steps

\subsubsection{Cluster head Selection}

In LEACH-C, $\mathrm{CH}$ selection mainly depends on the remaining energy and number of $\mathrm{CHs}$ in the network. In the proposed algorithm, the $\mathrm{CH}$ selection equation of LEACH-C had been extended by inserting the distance to BS and distance to neighbour nodes within the cluster (nodes with the same centroid). The sum of the distance to BS and the distance to neighbor nodes (intra + inter) represents the total communication cost distance when the concerned node is selected as $\mathrm{CH}$. Thus, a node with more residual energy and less communication distance will be more likely to become $\mathrm{CH}$ in its cluster. $\mathrm{CH}$ selection equations in (3) \& (4) can be rewritten as:

$$
\begin{gathered}
P_{i}(t)=\max \left\{\left(\frac{E_{i}(t)}{E_{\text {totata }}(t)}+\frac{1}{\text { comdis }_{i}{ }^{2}}\right), 1\right\}, \\
E[\# C H]=\sum_{i=1}^{n} p_{i}(t) * 1=\sum_{i=1}^{n}\left(\left(\frac{E_{1}(t)}{E_{\text {totali }}(t)}+\frac{1}{\operatorname{comdis~}_{1}{ }^{2}}\right)+\ldots+\left(\frac{E_{n}(t)}{E_{\text {totati }}(t)}+\frac{1}{\operatorname{comdis~}_{n}{ }^{2}}\right)\right) * 1=1
\end{gathered}
$$

where $\operatorname{comdis}_{i}{ }_{i}$ represents the communication distance of node $i$ and $n$ is the number of nodes in the cluster. Since the parameters $n, E_{i}(t), E_{\text {total }}(t)$ and $c o m d i s^{2}{ }_{i}$ are known, every node can calculate its probability. Generally, calculation cost is less than communication cost in terms of energy cost [10]. Then, the nodes with higher probabilities are more likely to become CHs in

their clusters. That means, the probability of being $\mathrm{CH}$ increases when a node has high energy and short communication distance among its neighbors. The probability ensures selection of optimal $\mathrm{CHs}$ in terms of entire energy consumption. The $\mathrm{CHs}$ advertize themselves and their cluster centroids, then member nodes belong to their $\mathrm{CHs}$ according to cluster centroids, that 
International Journal of Wireless \& Mobile Networks (IJWMN) Vol. 7, No. 1, February 2015

means, no need to excessive computations. Each $\mathrm{CH}$ determines its low level energy according to the number of its members and distance to BS, and then creates and sends TDMA schedule to its members. Also, every member node determines its low level energy according to the distance to its $\mathrm{CH}$.

\subsubsection{Multi-hop routing Paths Establishment}

The algorithm takes a mixture of single and multi-hop routing. The BS computes and broadcasts the average distance $d_{\text {avg }}$ to all nodes from the beginning, according to $d_{\text {avg }}=\frac{\sum_{i=1}^{N} d_{B S}}{N}$, where $d_{R S}$ is the distance between node $i$ and the BS. If the distance between $\mathrm{CH}$ and BS is less than or equal to the average distance, the $\mathrm{CH}$ communicates with $\mathrm{BS}$ directly. Otherwise, $\mathrm{CH}$ sends a message to other CHs including cluster head ID, distance to BS and energy. Neighbor CHs save this message at first, then feedback their own messages. Based on the feedback, the $\mathrm{CH}$ chooses a neighbor $\mathrm{CH}$ which has more residual energy and close to BS as its next hop node. Finally, all $\mathrm{CHs}$ find their parent nodes, keeping in mind that, the last parent node that directly communicates with BS sends data to BS without aggregation.

\subsection{Data Transmission Phase}

In this phase, data is received and aggregated by the $\mathrm{CHs}$, and then the $\mathrm{CHs}$ transmit data to parent $\mathrm{CHs}$ or to the BS. The phase consists of frames; in each frame all nodes send data to their $\mathrm{CHs}$ which in turn forward data to $\mathrm{BS}$. The $\mathrm{CHs}$ open their radios during the two phases, to receive joint request messages and send TDMA in setup phase and receive data from nodes in steady state phase. While, normal nodes are placed in to sleep mode, and every node opens its radio only in its own time-slot. Every node or $\mathrm{CH}$ checks its energy before sending data and saves its residual energy after sending data to $\mathrm{CH}$ or to the $\mathrm{BS}$. The data transmission phase is terminated only if the BS checks low level energy status from $\mathrm{CH}$ or member node. The system continues these rounds until every node's energy has been depleted.

\subsection{Cluster head Reselection and Cluster Reformation}

Every node checks its energy before sending data and saves its residual energy after sending data to $\mathrm{CH}$ or to the $\mathrm{BS}$. The selected $\mathrm{CH}$ will continue to work as cluster head until its energy level decreases to predefined value known as the low level energy. For any $\mathrm{CH}$, if the energy before sending data is below the predefined value (low level energy); the $\mathrm{CH}$ informs the $\mathrm{BS}$ via specific bit in the current data packet header. Energy status information (2 bit for 4 states: CH_ENERGYLOW, CH_ENERGYHIGH, MEMB_ENERGYLOW, MEMB_ENERGYHIGH) is piggybacked in a reserved field in the data packet header that is ready to be sent to the BS. The BS informs the entire network nodes about the starting of cluster head selection process at the next round. Then after receiving the last data packet of the current frame, the data transmission phase is terminated and the entire system moves to the cluster head selection step in the setup phase to select new cluster heads from the existing clusters. Since there are no dead nodes, cluster formation process during this stage may generate clusters similar to the current ones. The system continues these rounds until one of the member nodes energy decreases below its low level. The 
International Journal of Wireless \& Mobile Networks (IJWMN) Vol. 7, No. 1, February 2015

member node informs its corresponding $\mathrm{CH}$, which in turn informs the BS. Member node energy status information ( 1 bit for 2 states: MEMB_ENERGYLOW, MEMB_ENERGYHIGH) is piggybacked in a reserved field in the data packet header that is ready to be sent to the $\mathrm{CH}$. When some nodes died, the total number of nodes is decreased, hence, network topology and nodes distribution are changed. So, new cluster formation process is important to maintain the adaptability of the algorithm. Thus, if the BS checks low energy status from member node, the system moves to cluster formation step in setup phase to form new clusters. Recalling that, only $\mathrm{CH}$ selection is done when the BS checks low energy status from any $\mathrm{CH}$. The system continues these rounds until energy depletion. Figure 2 shows the flowchart of the proposed algorithm.

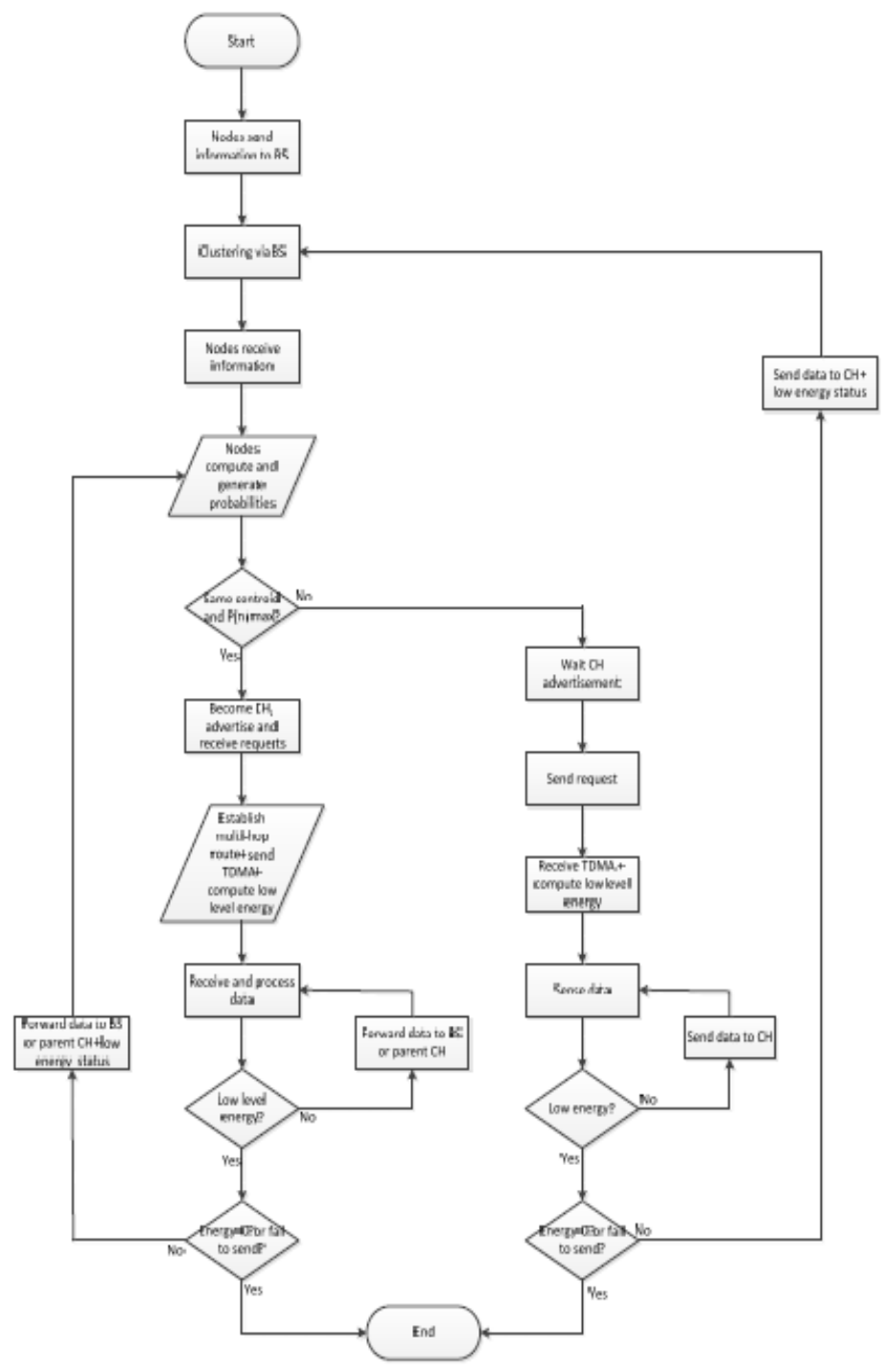

Figure 2. Flowchart of proposed algorithm 


\subsubsection{Calculation of Low level Energy for $\mathrm{CH}$}

The low level energy for $\mathrm{CH}$ is calculated after every process of cluster head selection. The low level energy is proportional to the number of member nodes of the $\mathrm{CH}$ and the distance of the $\mathrm{CH}$ from the BS.

If we assume that, the $\mathrm{CH}$ has $n$ member nodes and its distance from the $\mathrm{BS}$ is $\mathrm{d}_{\mathrm{CH}}$ then;

$$
E_{\text {resi }}=E_{\max }-E_{C H}
$$

where $\mathrm{E}_{\text {resi }}$ represents the residual energy of the $\mathrm{CH}, \mathrm{E}_{\max }$ is the initial energy or the residual energy of the $\mathrm{CH}$ after the previous sending process and $\mathrm{E}_{\mathrm{CH}}$ is the energy consumed by the $\mathrm{CH}$ to send a packet of data to the BS. The energy consumed by $\mathrm{CH}$ to receive, aggregate, and send a packet of data to the BS is given by;

$$
E_{C H}=E_{R X}+E_{D A}+E_{T X}
$$

where $\mathrm{E}_{\mathrm{RX}}$ represents the energy dissipated due to receiving data from member nodes, EDA is the aggregation energy, and ETX is the energy consumed by $\mathrm{CH}$ to transmit aggregated data to BS. Equation (9) can be rewritten as;

$$
\left\{\begin{array}{l}
E_{C H}=n l E_{\text {elec }}+(n+1)\left[l E_{D A}+l E_{\text {elec }}+l \varepsilon_{m p} d_{C H}^{4}\right] \\
E_{C H}=n l E_{\text {elec }}+(n+1)\left[l E_{D A}+l E_{\text {elec }}+l \varepsilon_{m p} d_{C H}^{2}\right]
\end{array}\right.
$$

Since $E_{\text {elec }}, E_{D A d}, k$ and $\varepsilon_{m v}$ are constants, then;

$$
\begin{gathered}
E_{C H} \propto n+(n+1)+(n+1) d_{C H}^{4} \\
\text { i.e., } E_{C H}=c_{1} n+c_{2}(n+1)+c_{3}(n+1) d_{C H}^{4}
\end{gathered}
$$

where $c_{1}=l E_{\text {elec }}, c_{2}=l E_{\text {elec }}+l E_{D A}$ and $c_{3}=l \varepsilon_{m p}$ then:

$$
\begin{gathered}
E_{C H}=n+(n+1) \frac{c_{2}}{c_{1}}+(n+1) \frac{c_{3}}{c_{1}} \Rightarrow n+(n+1)\left[\frac{E_{D A}}{E_{\text {elec }}}+1\right]+(n+1) \frac{\varepsilon_{m p}}{E_{\text {eiec }}} d_{C H}^{4} \\
\text { i.e., } \quad E_{C H}=n+(n+1)\left[1+\frac{E_{D A}}{E_{\text {eiec }}}+\frac{\varepsilon_{m p} d_{C H}^{4}}{E_{\text {eiec }}}\right]
\end{gathered}
$$

Assuming that $\frac{E_{D A}}{E_{\text {elec }}}=\alpha$ and $\frac{\varepsilon_{m p}}{E_{\text {elec }}}=\beta_{4}$;

$$
E_{C H}=n+(n+1)\left[1+\alpha+\beta_{4} d_{C H}^{4}\right]
$$


To ensure that the $\mathrm{CH}$ has enough energy to continue as $\mathrm{CH}$, the minimum energy required is:

$$
\begin{gathered}
E_{\max }>=2 E_{C H} \\
\text { i.e., } E_{\max }>=2\left(n+(n+1)\left[1+\alpha+\beta_{4} d_{C H}^{4}\right]\right)
\end{gathered}
$$

So, whenever a $\mathrm{CH}^{\prime} \mathrm{s} E_{\max }$ (energy before sending data) becomes less than $2\left(n+(n+1)\left[1+\alpha+\beta_{4} d_{C H}{ }^{4}\right]\right)$, the $\mathrm{CH}$ sets a specific bit in the current data packet header which is ready to be sent to the BS in the current TDMA frame. From (15) the predefined value (or low level energy) for $\mathrm{CH}\left(\lambda_{\mathrm{CH}}\right)$ can be defined as:

$$
\lambda_{C H} \begin{cases}<2\left(n+(n+1)\left[1+\alpha+\beta_{4} d_{C H}^{4}\right]\right), & \text { if } d>d_{0} \\ <2\left(n+(n+1)\left[1+\alpha+\beta_{2} d_{C H}^{2}\right]\right), & \text { if } d \leq d_{0}\end{cases}
$$

where $\alpha, \beta_{4}$ and $\beta_{2}$ are application specific constants such that $\alpha=\frac{E_{D A}}{E_{\text {elec }}}, \beta_{4}=\frac{\varepsilon_{m p}}{E_{\text {elec }}}$,

$\beta_{2}=\frac{\varepsilon_{f s}}{E_{\text {elec }}}, d_{C H}$ is the distance from the $\mathrm{CH}$ to the BS and $n$ is the number of member nodes which have the same centroid as the $\mathrm{CH}$. Then each $\mathrm{CH}$ determines its predefined value of the residual energy after every cluster head selection process according to the number of member nodes $(n)$ and the distance to $\mathrm{BS}\left(d_{C H}\right)$.

\subsubsection{Calculation of Low level Energy for normal node}

The low level energy for every node is adjusted after every process of cluster formation. The low level energy is proportional to the distance of member node from the $\mathrm{CH}$. If $\mathrm{d}_{\mathrm{n}}$ is the distance of the node from the $\mathrm{CH}$, then;

$$
E_{n \text { resi }}=E_{n \max }-E_{n}
$$

where $E_{\text {nresi }}$ represents the residual energy of the node, $E_{n \max }$ is the initial energy or the residual energy of the member nodde after the previous sending process and $E_{n}$ is the energy consumed by the node during sending data to the $\mathrm{CH}$. The energy consumed by member node to transmit data packet to its $\mathrm{CH}$ is given by;

$$
\begin{aligned}
& E_{n}=k E_{\text {elec }}+k \varepsilon_{f s} d_{n}^{2} \\
& \text { i.e., } E_{n}=1+\frac{\varepsilon_{f s}}{E_{\text {eler }}} d_{n}^{2}
\end{aligned}
$$


where $\frac{\varepsilon_{f s}}{E_{\text {elec }}}=\beta_{2}$ and $d_{n}$ is the node distance to the $\mathrm{CH}$. To ensure that the node has enough

energy to continue sensing and sending data to the $\mathrm{CH}$, the minimum energy required is:

$$
\begin{gathered}
E_{n \max }>=2 E_{n} \\
\text { i.e., } E_{n \max }>=2\left(1+\frac{\varepsilon_{f s}}{E_{\text {elec }}} d_{n}^{2}\right) \Rightarrow 2\left(1+\beta_{2} d_{n}^{2}\right)
\end{gathered}
$$

So, when the energy of the node (before sending data to $\mathrm{CH}$ ) becomes less than $2\left(1+\beta_{2} d_{n}^{2}\right)$, the node sets specific bit in the current sensed data packet header which is ready to be sent to the $\mathrm{CH}$ in the current slot. Accordingly, the $\mathrm{CH}$ notifies the $\mathrm{BS}$ and the system moves to form new clusters. From (19) the low level energy value for normal node can be defined as:

$$
\lambda_{n}\left\{\begin{array}{l}
<2\left(1+\beta_{2} d_{n}^{2}\right), \text { if } d \leq d_{0} \\
<2\left(1+\beta_{4} d_{n}^{4}\right), \text { if } d>d_{0}
\end{array}\right.
$$

where $\beta_{2}, \beta_{4}$ are as defined before and $d_{n}$ is the node distance to the $\mathrm{CH}$.

\section{SIMULATIONS AND RESULTS}

The simulation environment consists of 100 nodes deployed randomly over a sensing field of 100100 meters; the BS is located inside the sensing field. The control and data message lengths are 200bits and 6400 bits respectively, with aggregation factor $c$ set to unity, such that every node has data to send in each round. The initial energy of each node is $0.5 \mathrm{~J}$ and the other simulation parameters are as explained in the network energy model. In this work the following metrics are used to evaluate the life time and performance of the network; time taken till all nodes die, data sent to BS and the time taken till the first node die (stability period). 


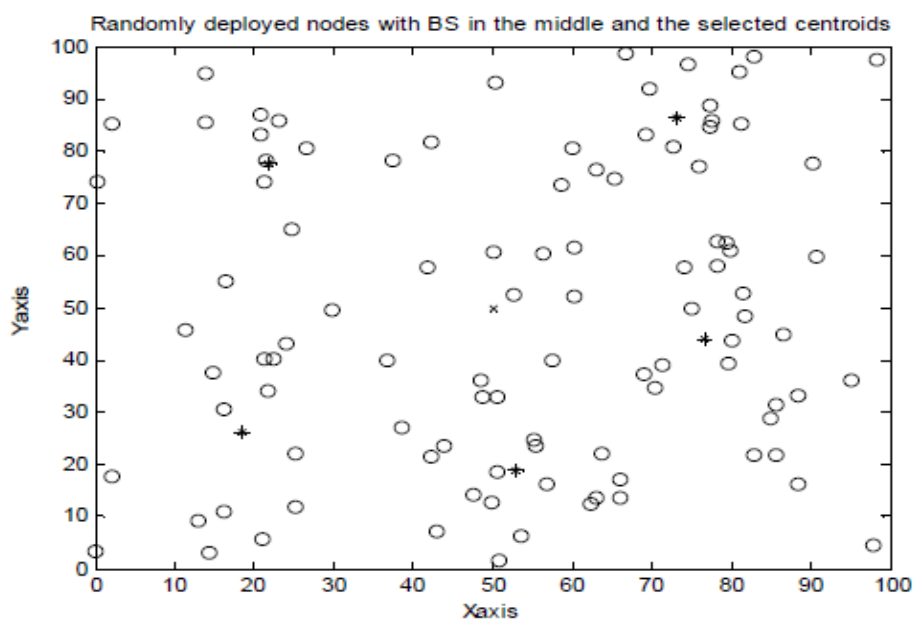

Figure 3. Sensing field including BS and centroids

Figure 3 shows the centroids of clusters obtained by k-mean clustering method at the beginning of the simulation. The small circles are the deployed nodes and the black star marks are the cluster centroids after cluster formation, while the cross mark in the middle is the BS.

Table 1. Simulation results

\begin{tabular}{|l|l|l|l|l|}
\hline Algorithm & Dead nodes & Stability Period & Rounds & Data sent to BS \\
\hline LEACH-C & 100 & 101 & 292 & 1543 \\
\hline $\begin{array}{l}\text { Proposed } \\
\text { Algorithm }\end{array}$ & 100 & 221 & 700 & 2265 \\
\hline
\end{tabular}

Table 1 shows the dead nodes, stability period, life time and the data sent to BS for LEACH-C and the proposed algorithm as obtained by the simulation. From Figure 4 and table 1, the network life time and the stability period for proposed algorithm are both twice more as the network life time and the stability period for LEACH-C. There is a significant improvement in the network life time and performance due to the clustering technique, minimum communication cost $\mathrm{CH}$ selection technique and on demand clustering. The minimum energy clustering technique optimizes the distance between $\mathrm{CHs}$ and members reducing energy consumption and changes of signal strength within cluster. The $\mathrm{CH}$ selection technique selects the optimized node as head node, which has the minimal cost in terms of energy while communicating with others. 


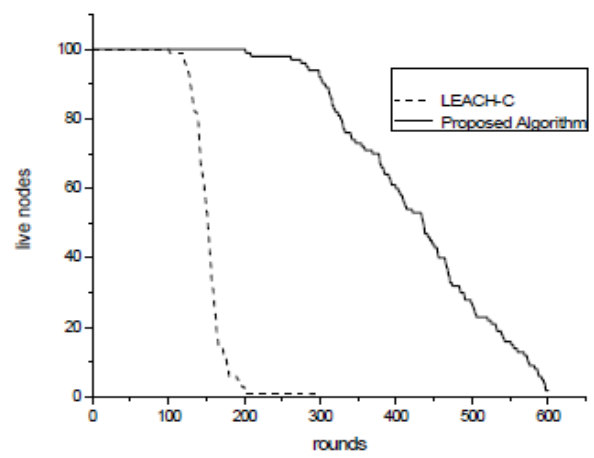

Figure 4. Live nodes vs. rounds

Moreover, the low level energy technique permits the previous $\mathrm{CHs}$ to function as normal nodes in the consecutive rounds and avoids sudden death of $\mathrm{CHs}$, hence, loss of data. Consequently, the data sent to BS is increased, improving the network reliability in comparison to LEACH-C. Therefore, in proposed approach the network does not exhaust energy suddenly and quickly, resulting in long stability period.

Figure 5 shows the increase in the total data sent to BS; it is increased approximately by $47 \%$ than LEACH-C as shown in table 1 . The reason is that, the life time of the network is extended in the proposed algorithm such that it survives for longer time than in LEACH-C, in addition to the effect of low energy technique.

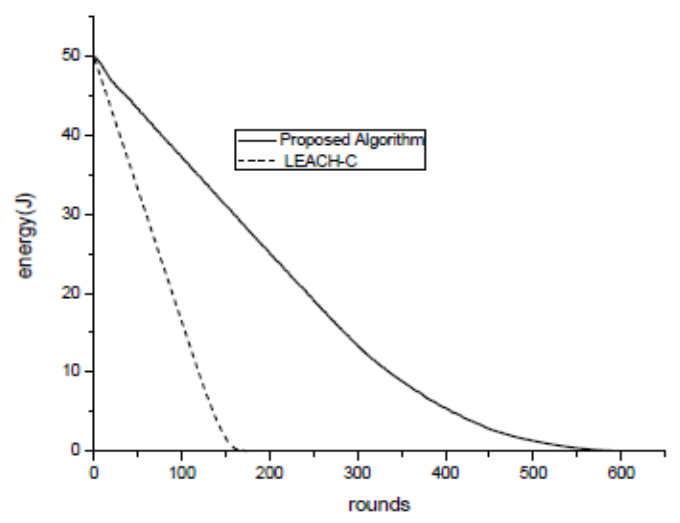

Figure 5. Energy vs. rounds

Figure 6 and Figure 7 show the variation of the network energy, in the proposed algorithm the nodes didn't run out of energy suddenly and fast. That occurred, because the former cluster head will function as normal node when new cluster head is selected. By not performing the clustering process in each round, the number of clustering processes in proposed algorithm is much less than this value in LEACH-C. 


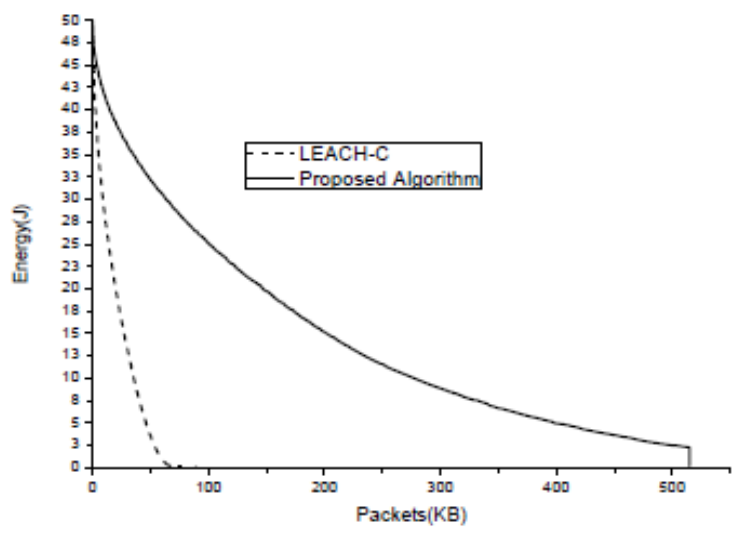

Figure 6. Energy vs. packets

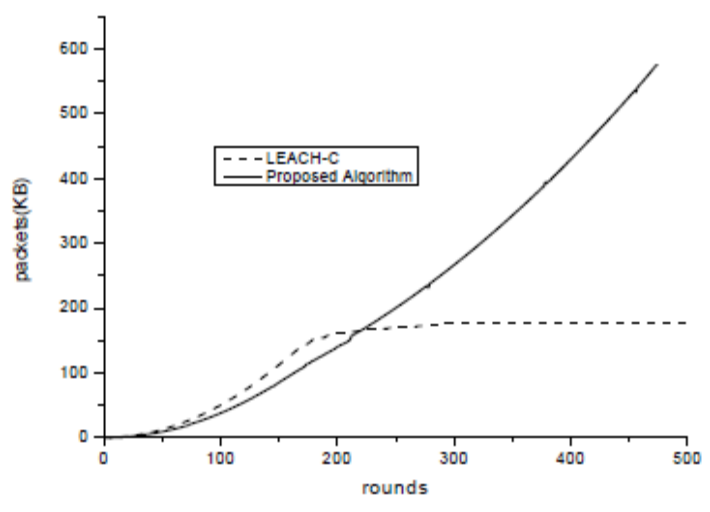

Figure 7. Packets vs. rounds

The protocol performs only cluster head selection from the existing clusters, and starts forming new clusters in the subsequent rounds when any member node consumes predefined value of its energy. Hence, the protocol avoids the time and energy consumed for periodic or dynamic clustering in each round. That means, after the first setup phase, the clustering will not be performed until at least one of the $\mathrm{CHs}$ or member nodes consumes a predefined value of its energy, because doing clustering at the beginning of each round imposes lots of overhead on the network. This in turn, decreases the overhead caused by periodic clustering in each round. The BS and the nodes are stationary, and every $\mathrm{CH}$ sends its cluster energy with every data packet, i.e. the BS updates the value of total energy continuously. So, the nodes need to send their location and energy information directly to the BS only once (at the beginning of working) during the network life time. This introduces dramatic improvement in the entire network energy consumption, hence, in network life time.

Finally, most important and critical issue controlled by the low level energy, is the length of the steady-state period, because how to set the time length of each round to prolong the life time and increase the throughput is very critical. The length of the steady-state period is critical to achieve 
International Journal of Wireless \& Mobile Networks (IJWMN) Vol. 7, No. 1, February 2015

the energy reduction necessary to offset the overhead caused by cluster formation and cluster head selection processes. Short steady-state period increases the protocol's overhead, where as long period may lead to cluster head energy depletion and loss of data. In this approach, no need to define the data transmission period in advance (define super frame) or do periodic clustering, the algorithm terminates data transmission phase automatically before the transmitting node energy depletion, thus protects the network from loss of data. In the proposed algorithm, by increasing the number of rounds the number of clustering processes increases progressively. The reason is that by decreasing energy of nodes, the number of reclustering that should be held increases. From the above results, the proposed algorithm prolongs the life time and the stability period of the network, hence, improves the energy and performance efficiency of the wireless network.

\section{CONCLUSIONS}

The transmission distance and control overhead have a great impact on energy consumption, as well as, the regular consumption of the energy over the entire network. So, a technique that minimizes cluster energy, and a method that optimizes $\mathrm{CH}$ section in each cluster had been used in order to balance energy consumption over the entire network. Furthermore, minimum energy paths were applied to forward data in a multi-hop routing to conserve more energy. The usage of minimum energy clustering technique led to formation of well distributed, compact, and minimum energy clusters that avoid creation of network holes and existence of excluded nodes. On demand clustering enables the approach to vary the duration of data transmission phase automatically according to node energy status. Therefore, reduces the periodic clustering overhead and the chances of data loss due to $\mathrm{CH}$ energy depletion during data transmission phase. The simulation results show that the algorithm balances the load among nodes, prolongs the stability period and life time of the network, and improves network reliability compared with LEACH-C.

Although the results confirmed that the proposed algorithm outperforms LEACH-C, there are many other protocols that have to be compared. Also, since k-mean is iterative clustering algorithm, it is significantly sensitive to initial parameters. So, a good selection of initial parameters is an essential clustering problem in order to shorten the convergence time. Also, since sensors are deployed randomly, irregular density problem is expected, so how to control the number of associated cluster members in each cluster or how to create relatively load balanced clusters such that no cluster head is heavily loaded regardless of its proximity to the sink, is interesting research direction. Furthermore, the approach can be generalized via coordinators or gateways to be suitable for large scale applications. These issues will be our future study directions. 
International Journal of Wireless \& Mobile Networks (IJWMN) Vol. 7, No. 1, February 2015

\section{REFERENCES}

[1] K. Akkaya and M.Younis, (2005), "Asurvey of Routing Protocols in Wireless Sensor Networks", Elsevier Ad Hoc Network Journal, Vol. 3/3, pp. 325-349.

[2] C. Shilpa, Dr. M. Sona and C. Yogesh, (July 2012)"Asurvey of Hierarchical Routing Protocols in Wireless Sensor Networks", International Journal of Emerging trends in Engineering and Development, ISSN 2249-6194, Issue 2, Vol.5

[3] I. F. Akyildiz and M. C. Vuran, (2010) "WSN applications," Wireless Sensor Networks, pp. 17-35.

[4] G. Anastasi, M. Conti, M. Di Francesco, and A. Passarella, (2009) "Energy Conservation in wireless sensor networks: a survey," Ad Hoc Networks, vol. 7, no. 3, pp. 537-568.

[5] Y. Ha, H. Kim, and Y. Byun, (2012) "Energy-efficient fire monitoring over cluster-based wireless sensor networks," International Journal of Distributed Sensor Networks, vol. 2012, Article ID 460754, 11 pages.

[6] Hyunjo Lee, Miyoung Jang, and Jae-Woo Chang , (2014)“A New Energy-Efficient Cluster-Based Routing Protocol Using a Representative Path in Wireless Sensor Networks" International Journal of Distributed Sensor Networks, vol. 2014, Article ID 527928, 12 pages http://dx.doi.org/10.1155/2014/527928

[7] Sanjeev Kumar Gupta, Neeraj Jian, Poonam Sinha, (Jan 2013)" Clustering Protocols in Wireless Sensor Networks: A Survey", International Journal of Applied Information Systems (IJAIS)-ISSN: 2249-868, FCS, New York, USA, vol. 5, no. 2, pp. 41-50,www.ijais.org.

[8] Xuxun Liu and Jinglun Shi (July 2012)" Clustering Routing Algorithm In Wireless Sensor Networks: An Overview", KSII transactions on Internet and Information Systems, vol. 6, no. 7, pp. 1735-1755.

[9] Prakashgoud Patil, Umakant Kulkarni, N. H. Ayachit, (2011)" Some Issues in Clustering Algorithms for Wireless Sensor Networks", IJCA Special Issue on "2nd National Conference-Computing, Communication and Sensor Network" CCSN, pp. 18-23. .

[10] W. B. Heinzelman, A. P. Chandrakasan, and H. Balakrishnan, (2002) "Application-specific protocol architecture for wireless micro sensor Networks," IEEE Transactions on Wireless Communications, vol. 1, no. 4, pp. 660-670.

[11] Fan, X.; Song, Y. October (2007) "Improvement on LEACH Protocol of Wireless Sensor Network", In Proceedings of International Conference on Sensor Technologies and Applications, Valencia, Spain, 14-20, pp. 260-264.

[12] M.O. Farooq, A. B. Dogar, and G.A. Shah, (July 2010) "MR-LEACH: multi-hop routing with low energy adaptive clustering hierarchy, "in Proceedings of the 4th International Conference on Sensor Technologies and Applications (SENSORCOMM '10), pp. 262-268, Venice, Italy.

[13] Z. Yong and Q. Pei, (2012) "An energy efficient clustering routing algorithm based on distance and residual energy for wireless sensor networks,"Procedia Engineering, vol. 29, pp. 1882-1888.

[14] N. Javaid, T. N. Qureshi, A. H. Khan, A. Iqbal, E. Akhtar, and M. Ishfaq, (2013) "EDDEEC: enhanced developed distributed energy efficient clustering for Heterogeneous wireless sensor NETworks," Procedia Computer Science, vol. 19, pp. 914-919.

[15] Osama Younis, Sonia Fahmy, (October-December 2004)"HEED: Ahybrid, Energy Efficient, Distributed Clustering approach for Ad Hoc Sensor Networks" IEEE transactions on Mobile Computing, vol. 3, No.4, pp. 366-379.

[16] Vevik Katiyar, Narottan Chand, (March 2011)'Improvement in LEACH Protocol for large -scale WSNs", Proceedings of (ICETECT), Tamil Nadu, pp.1070-1075.

[17] S. A. Nikolidakis, D. Kandris, D. D. Vergados, andC. Douligeris, (2013) "Energy efficient routing in wireless sensor networks through balanced clustering," Algorithms, vol. 6, no. 1, pp. 29-42.

[18] Chunyao FU, Zhifang JIANG, Wei WEI, Ang WEI, (Jan 2013) "An Energy Balanced Algorithm of LEACH protocol in WSNs", IJCSI International Journal of Computer Science Issues, vol. 10, Issue. 1, no. 1, pp. 354-359.

[19] Jian Xu, Dandan Qin, (2013) “A New LEACH-Based Routing Clustering Protocol in WSNs”, Journal of Information \& Computational Science, pp. 6005-6011. www.joics.com 
International Journal of Wireless \& Mobile Networks (IJWMN) Vol. 7, No. 1, February 2015

[20] R. P. Pantonia and D. Brandao, (2013) "A gradient based routing scheme for street lighting wireless sensor networks," Journal of Network and Computer Applications, vol. 36, no. 1, pp. 77-90.

[21] F. Ren, J. Zhang, Y. Wu, T. He, C. Chen, and C. Lin, (2013) "Attribute aware data aggregation using potential-based dynamic routing in wireless sensor networks," IEEE Transactions on Parallel and Distributed Systems, vol. 24, no. 5, pp. 881-892.

[22] Tadahiko Murada and Hisao Ishibuchi, (June 1994) "Evaluation of Genetic Algorithms flow shop scheduling Problems", proc. 1st IEEE Conference Evolutionary computation, vol. 2, pp. 812-817.

[23] Zhengxin Chen, (2001) "Data Mining and Uncertain Reasoning-An Integrated approach", John Willey, ISBN 0471388785.

[24] J.-S., Jang, Sun, C.-T. and Mazutani, (1997) "Neuro-Fuzzy and Soft Computing: a computational approach to learning and machine intelligence," ISBN 0-13-261066-3, Prentice Hall, Upper Saddle River, NJ, USA. 